\title{
A COTS RF/Optical Software Defined Radio for the Integrated Radio and Optical Communications Test Bed
}

\author{
Jennifer M. Nappier ${ }^{1}$, Daniel J. Zeleznikar ${ }^{2}$, Adam C. Wroblewski ${ }^{3}$, Roger P. Tokars ${ }^{4}$, Bryan L. Schoenholz ${ }^{5}$ and \\ Nicholas C. Lantz \\ NASA Glenn Research Center, Cleveland, Ohio 44135
}

\begin{abstract}
The Integrated Radio and Optical Communications (iROC) project at the National Aeronautics and Space Administration (NASA) is investigating the merits of a hybrid radio frequency (RF) and optical communication system for deep space missions. In an effort to demonstrate the feasibility and advantages of a hybrid RF/Optical software defined radio (SDR), a laboratory prototype was assembled from primarily commercial-off-the-shelf (COTS) hardware components. This COTS platform has been used to demonstrate simultaneous transmission of the radio and optical communications waveforms through to the physical layer (telescope and antenna). This paper details the hardware and software used in the platform and various measures of its performance. A laboratory optical receiver platform has also been assembled in order to demonstrate hybrid free space links in combination with the transmitter.
\end{abstract}

\section{Nomenclature}

$K_{S} \quad=$ signal photon counts per signal slot

$K_{b} \quad=$ background photons per slot

\section{Introduction}

$\mathrm{T}$ HE Integrated Radio and Optical Communications (iROC) project ${ }^{1}$ has a goal of researching and developing technology to support a hybrid radio frequency (RF) and optical communication system for National Aeronautics (NASA) deep space missions. These technology areas include beaconless pointing ${ }^{2}$, a combined RF and optical aperture, and an RF and optical software defined radio (SDR). Within the radio, the RF and optical systems could be integrated to varying degrees from a fully integrated custom application specific integrated circuit (ASIC) to separate radios managed by a common processor. The architecture presented here utilizes commercial-off-the-shelf (COTS) components and seeks to take advantage of the reconfigurable nature of the SDR by integrating the RF and optical waveforms onto one field programmable gate array (FPGA) with separate mezzanine cards for the RF and optical transmitters.

This paper describes the transmitter architecture, including the RF and optical SDR, in Section II. Testing results for both the RF and optical transmitters are described in Section III. Conclusions and future work are presented in Section IV.

\footnotetext{
${ }^{1}$ Aerospace Technologist in Data Systems, Information and Signal Processing Branch, 21000 Brookpark Road MS 54-1.

${ }^{2}$ Aerospace Technologist in Data Systems, Information and Signal Processing Branch, 21000 Brookpark Road MS 54-1.

${ }^{3}$ Research Aerospace Technologist in Measurement and Instrument Systems, Optics and Photonics Branch, 21000 Brookpark Road MS 77-1.

${ }^{4}$ Research Aerospace Technologist in Electro-Optical Sensor Systems, Optics and Photonics Branch, 21000 Brookpark Road MS 77-1.

${ }^{5}$ Aerospace Technologist in Telecommunications, Advanced High Frequency Branch, 21000 Brookpark Road MS 54-1.

${ }^{6}$ Aerospace Technologist in Avionic Systems, Avionics Branch, 21000 Brookpark Road MS 309-2.
}

American Institute of Aeronautics and Astronautics 


\section{Transmitter Architecture}

The transmitter architecture can be broken down into four subsystems. They are the SDR Prototype, the RF Subsystem, the Electro Optic Subsystem, and the Optics Subsystem. A block diagram of the system can be seen in Figure 1.

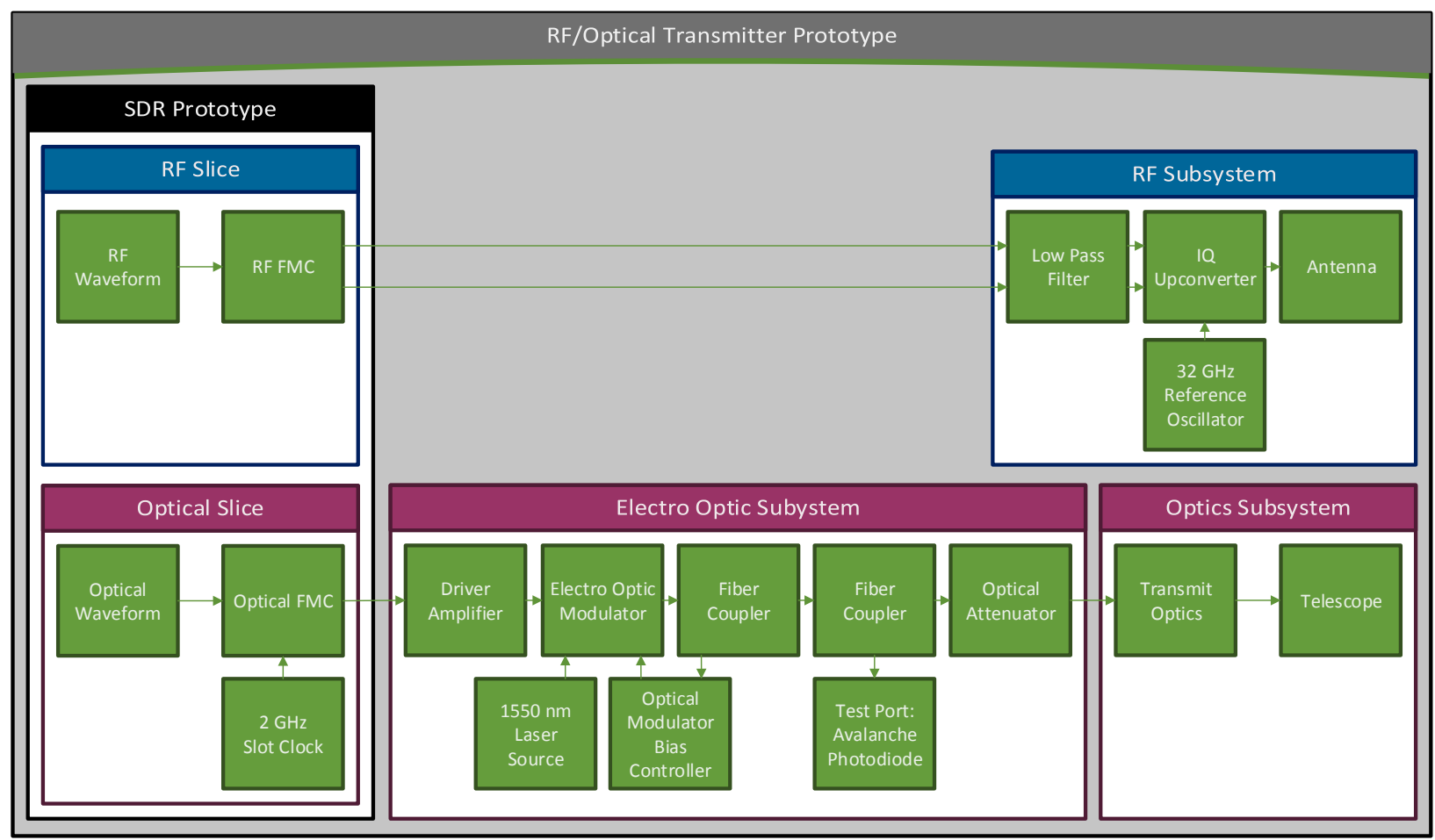

Figure 1. Transmitter Architecture

\section{A. Software Defined Radio Prototype}

The SDR performs the waveform generation for the RF and optical signals. The digital processing platform for the SDR is a Xilinx FPGA development board. The development board is configured to automatically program the FPGA with the RF and optical waveform firmware at power-up. Both the RF and the optical waveform run simultaneously on the FPGA. The development board includes two FPGA Mezzanine Card (FMC) interfaces: one low-pin-count (LPC), and the other a high-pin-count (HPC). The LPC connector provides an interface to the COTS digital to analog converter circuit board used for the RF transmitter waveform. The HPC connector provides an interface to a custom parallel to serial circuit board used for the optical transmitter waveform.

1. Optical Slice

The optical waveform implemented on the FPGA development board is serially concatenated pulse position modulation $^{4}$ (SCPPM). This waveform was designed by the Jet Propulsion Laboratory (JPL) for use in a Mars mission and was also used on the Lunar Laser Communications Demonstration ${ }^{3}$ (LLCD) in 2013. The SCPPM waveform Verilog code received from JPL is PPM-16 with a rate $1 / 2$ code. Four slots of inter symbol guard time and a 16 symbol frame acquisition sequence (FAS) were added to the waveform. A block diagram of the waveform can be seen in Figure 2. The controller block generates data at the correct intervals for the $1 / 2$ rate SCPPM modulator. The data generator consists of a pseudo-random bit stream. The FAS append block adds the 16 symbol frame acquisition sequence. The PPM-16 symbol to slot mapper block maps the 4 bit symbols to 16 bit slots and the guard time insertion block adds 4 slots of guard time. The slots are read out of the FIFO by the waveform controller and converted from 20 parallel bits to the 16 bit parallel interface which is compatible with the optical FMC. 


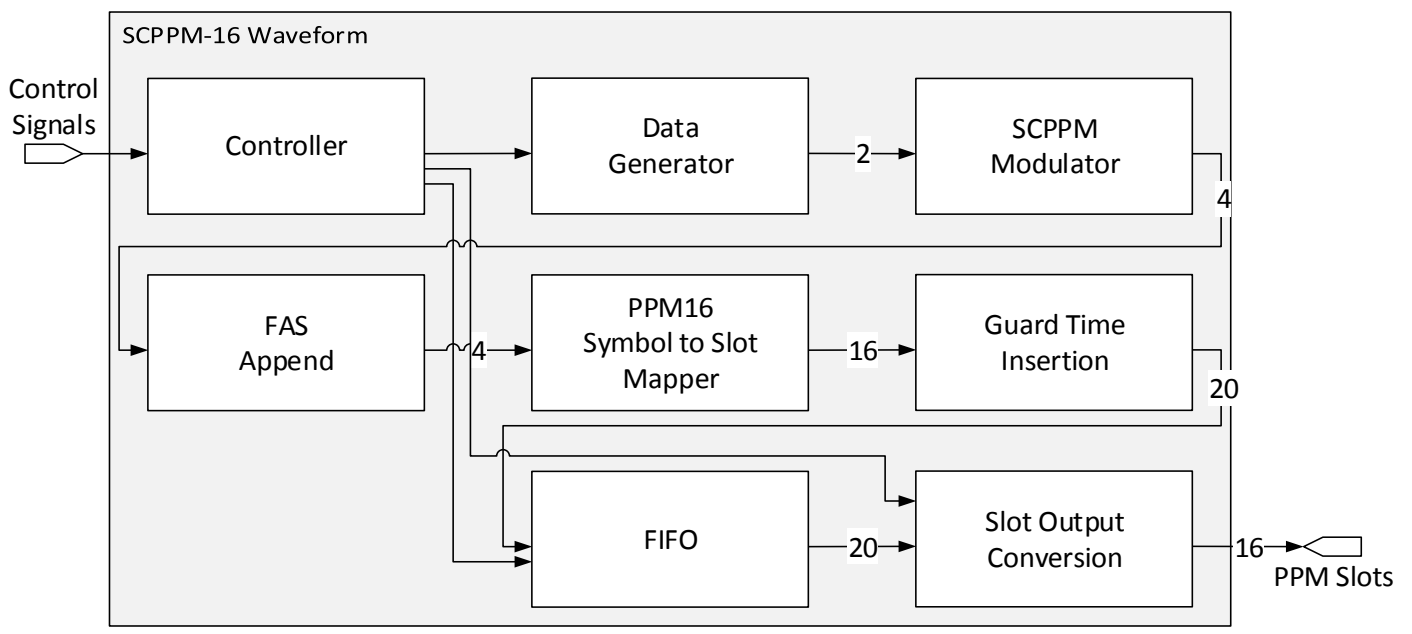

Figure 2. SCPPM Waveform Block Diagram.

The optical FMC performs parallel to serial conversion for the SCPPM encoded data. A slot clock signal is provided to the optical FMC at rates up to $2 \mathrm{GHz}$. This clock rate is used directly by the optical FMC for serial data output. This clock signal is divided by $16(125 \mathrm{MHz}$ if using a $2 \mathrm{GHz}$ slot clock) and fed back to the FPGA. The FPGA supplies 16 parallel signals to the optical FMC at this slower clock rate. This clock division method ensures that the parallel data is updated at the precise time needed by the parallel to serial conversion circuit. The main advantage of this approach is that it confines high speed signaling to a small subset of components. This results in a simpler, more robust hardware design.

2. RF Slice

The RF waveform selected is pulse-shaped Gaussian Mean Shift Keying (GMSK) or offset quadrature phase shift keying (OQPSK) with low density parity check codes (LDPC) or Turbo codes 5 . For the initial laboratory demonstration of the hybrid communications system, only the OQPSK modulation was implemented on the Xilinx FPGA development board. In the future, the full waveform will be implemented on the Xilinx FPGA development board. The waveform ${ }^{6}$ was originally demonstrated on the Space Communication and Navigation (SCaN) Test bed ${ }^{7}$.

The RF FMC consists of the digital to analog converter (DAC) and the analog digital converter (ADC) card. This card includes an on-board oscillator at a frequency of 737.28 MHz which drives both the waveform firmware on the FPGA, and the DAC. The clock divider chip on the FMC divides the clock by four down to $184.32 \mathrm{MHz}$ to drive the RF waveform in the FPGA. The OQPSK waveform is sampled at a rate of 4 samples/symbol to give a symbol rate of 46.08 MSPS.

\section{B. Electro Optic Subsystem}

The electro optic subsystem takes as an input the electrical PPM pulses coming out of the optical FMC and converts them into optical pulses of light at the $1550 \mathrm{~nm}$ wavelength using a lithium niobate electro optic (EO) modulator. The driver amplifier converts the voltage level which is output from the optical FMC to the voltage which is necessary to drive the $\mathrm{EO}$ modulator. This amplification is especially necessary to achieve the maximum extinction ratio of the EO modulator.

The lithium niobate EO modulator is sensitive to environmental factors such as temperature and humidity and so the output must be controlled using a bias control feedback circuit. A fiber optic splitter is used to send 10\% of the transmitted light to a photo-detector on board the bias controller. The controller adjusts the bias voltage pin on the EO modulator as necessary to minimize the output power of the modulator.

The last few components in the optical transmitter train are present to provide key laboratory test features. The first component is a high speed photo-detector at one output of a second fiber-optic splitter as a test port. This test port can also be used to provide a measure of the extinction ratio of the modulator and to verify the correct waveform output. The final component of the system provides precise control of the transmitted optical power level in the form of an optical attenuator. This allows the system to emulate the Mars-Earth link, providing power levels to the receiver in the photon-counting regime.

American Institute of Aeronautics and Astronautics 


\section{Optics Subsystem}

A transmitter prototype, illustrated in Figure 3, has been assembled to transmit a modulated laser communication signal using COTS components. This prototype is being used to explore optical concepts based on the iROC design which utilizes a Cassegrain style transmitter. The optics in the transmitter are specifically chosen to maximize laser communication beam transmission power, in particular, to minimize communication beam blockage due to the Cassegrain's secondary reflector.

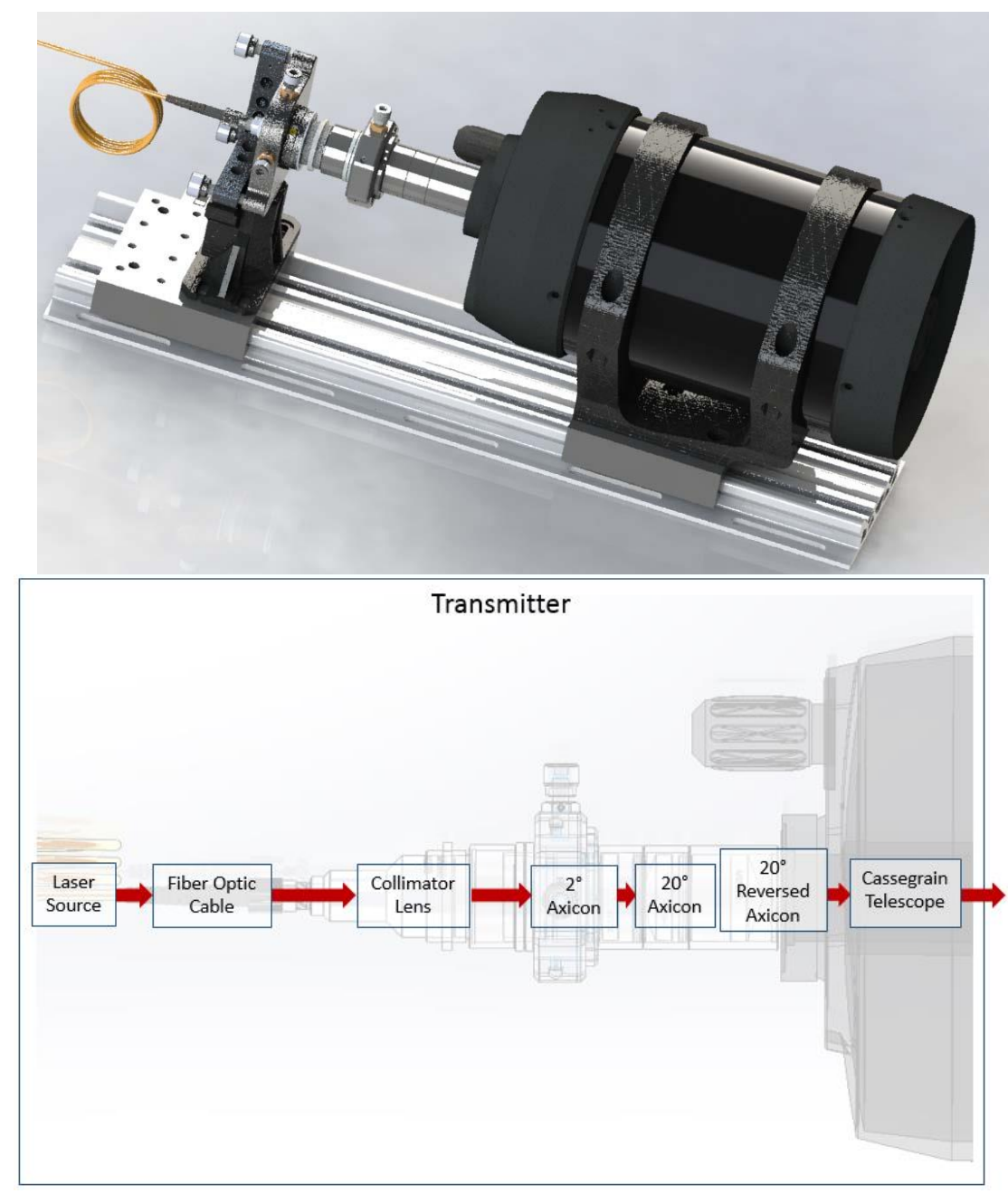

Figure 3. iROC transmitter rendered in 3D.

The optical fiber from the electro optic subsystem is coupled to a fiber launch collimating optic which directs the laser light to a series of axicon lenses. Axicon lenses have aspherical surfaces with a small radius of curvature and large conic constant specifically designed to generate an approximation of a Bessel beam in the form of a ring (Figure 4). This allows for directing nearly all the laser power around the secondary reflector of the Cassegrain at the exit of the aperture. The $2^{\circ}$ axicon generates the desired size and angle divergence necessary for the ring to pass through the geometry of the Cassegrain telescope. This axicon by itself must be placed far away (greater than 12 inches) from the Cassegrain entrance in order for the ring to be of sufficient size. The setup was compacted by using two additional $20^{\circ}$ axicons in series with opposite orientation in order to increase the ring size in a short distance. The optical components could then be placed right next to the entrance of the Cassegrain with tubing that enclosed all the optical components. 


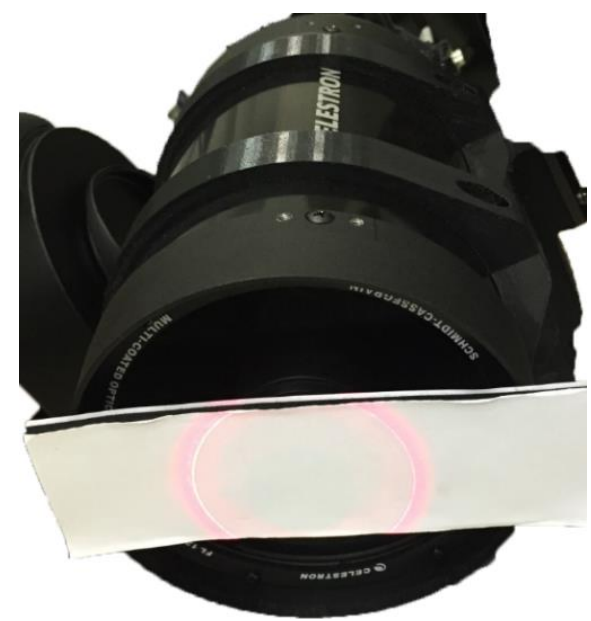

Figure 4. Axicon generates a laser ring to efficiently pass through the Cassegrain telescope.

\section{RF Subsystem}

The RF Subsystem filters and up-converts the in-phase and quadrature baseband signals to $32 \mathrm{GHz}$ for transmission over the air. The outputs from the RF FMC DACs are first filtered using a low pass filter to remove any unwanted signal images and unwanted clock signals that might come out of the DAC ports. These outputs are sent to a quadrature mixer which receives a precision oscillator input at $32 \mathrm{GHz}$ and mixes the baseband quadrature components directly to a center frequency at $32 \mathrm{GHz}$. Once mixed to this intended frequency for transmission, the output port can be connected to a test antenna with appropriate coaxial cabling.

\section{Results}

\section{A. Optical Results}

The optical SCPPM-16 transmitted signal was sent into an optical receiver post processing system ${ }^{8}$ for demodulation and decoding. The transmitter was configured for a $50 \mathrm{~ns}$ slot clock and a 2 Mbps data rate. The link was setup indoors in a clean room high bay. The receiver single photon counting detector was a photo multiplier tube and it was run at a supply of $575 \mathrm{~V}$ with a maximum current draw of close to $0.9 \mu \mathrm{A}$. 43,900 code words (166 seconds) were transmitted and decoded. Initially, a lower extinction ratio (specified at $20 \mathrm{~dB}$ ) optical modulator was used in testing, but this gave higher than desired background light levels, so it was replaced by a high extinction ratio (specified at $>40 \mathrm{~dB}$ ) electro optic modulator. The results show that $\mathrm{K}_{\mathrm{b}}$ decreased by one order of magnitude from 0.037 to 0.0025 and $\mathrm{K}_{\mathrm{s}}$ increased from 3.6 to 4.3 photon counts per signal slot. The code word errors decreased from 14 to 4 errors.

Table 1.Optical Link Testing Results.

\begin{tabular}{|l|l|l|l|l|l|}
\hline $\begin{array}{c}\text { Coded Words } \\
\text { Processed }\end{array}$ & $\begin{array}{c}\text { Code Word } \\
\text { Errors }\end{array}$ & $\begin{array}{c}\text { 信 } \\
\text { (photons/signal } \\
\text { slot) }\end{array}$ & $\begin{array}{c}\mathbf{K}_{\mathbf{b}} \\
\text { (photons/slot) }\end{array}$ & $\begin{array}{c}\text { Average PMT } \\
\text { Current }(\boldsymbol{\mu A} \text { A) }\end{array}$ & $\begin{array}{c}\text { Specified } \\
\text { Electro Optic } \\
\text { Modulator } \\
\text { Extinction } \\
\text { Ratio }\end{array}$ \\
\hline 43,900 & 14 & 3.6 & 0.037 & 0.91 & $20 \mathrm{~dB}$ \\
\hline 43,900 & 4 & 4.3 & 0.0025 & 0.93 & $>40 \mathrm{~dB}$ \\
\hline
\end{tabular}

\section{B. RF Results}

The RF transmit signal was evaluated just before the transmit antenna to measure its spectrum and modulation quality. A Keysight PXA Signal analyzer was used to take measurements directly at the transmit frequency of 32 GHz. Figure 5 shows the RF transmit signal spectrum. The spectrum has a slight dip near the center frequency which is likely from excess loss on the baseband signals near DC. This loss can be attributed to the DAC's AC coupled output.

American Institute of Aeronautics and Astronautics 


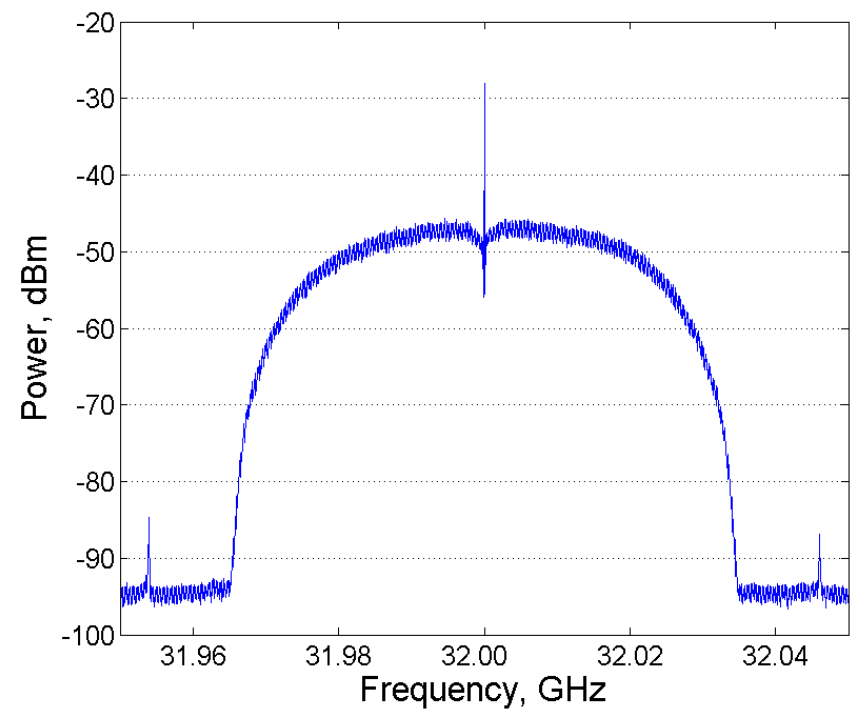

Figure 5. RF Transmit Signal Spectrum.

The Keysight Vector Signal Analysis software was used to digitally demodulate the OQPSK signal and make modulation quality measurements. Table 2 lists the measurement results. A constellation diagram can be seen in Figure 6 and the eye diagrams for the in-phase and quadrature signals is shown in Figure 7. As noted above, the losses in the baseband signals near DC cause signal distortion. This can be observed in the error vector measurement (EVM) which is reported to be $11.673 \% \mathrm{rms}$. A low convergence equalizer was used in the software to compensate for the frequency tilt of cables and the low pass filters. The Offset Error Vector Magnitude measurement accounts for only one point per symbol. The I-value from the beginning of each symbol and the $\mathrm{Q}$ value from the middle of each symbol are combined into a single complex value and compared to the ideal reference for this computation. In order to best detect these points the $\mathrm{Q}$ data is shifted by $1 / 2$ symbol. This shift helps line up decision points for the measurement however it also produces zero crossings in the data which results in a misleading trajectory between symbols in the constellation plot shown in Figure 6. The modulation quality could be improved by flattening out the frequency response of the DAC near DC and by tuning the baseband signal offsets to increase carrier suppression. Although the signal performance could be improved these measurements give confidence that the transmitter is functioning as designed and with a functional OQPSK receiver a data link could be established.

Table 2.RF Transmit Signal Modulation Quality Testing Results.

\begin{tabular}{|l|l|l|}
\hline Description & Value & Units \\
\hline Offset Error Vector Magnitude & 10.373 & $\% \mathrm{rms}$ \\
\hline Error Vector Magnitude & 11.105 & $\% \mathrm{rms}$ \\
\hline Phase Error & 6.520 & $\mathrm{Deg}$ \\
\hline Frequency Error & -15.158 & $\mathrm{~Hz}$ \\
\hline IQ Offset & -12.719 & $\mathrm{~dB}$ \\
\hline Quadrature Error & 4.116 & Deg \\
\hline Gain Imbalance & -0.748 & $\mathrm{~dB}$ \\
\hline
\end{tabular}




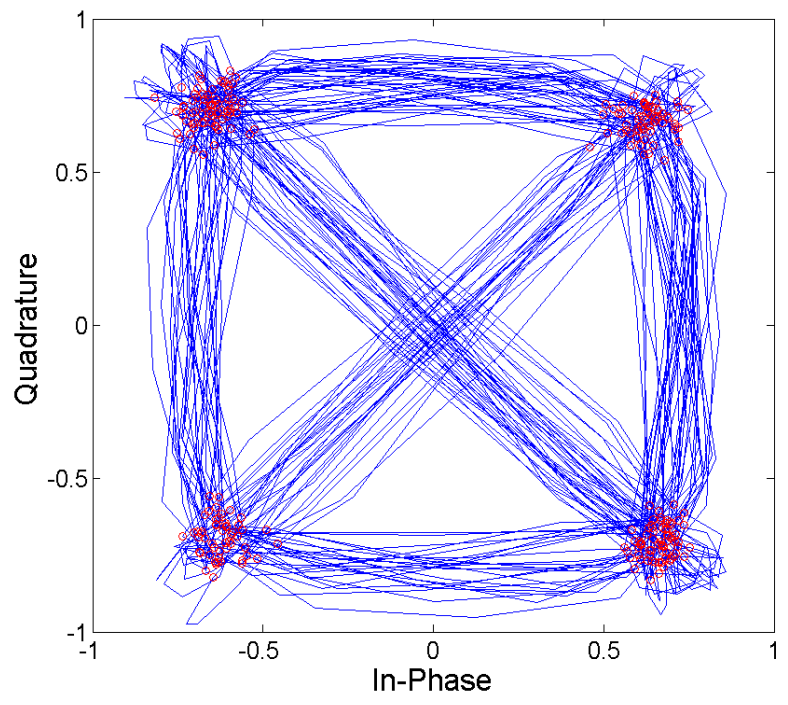

Figure 6. RF Transmit Signal Constellation Diagram.

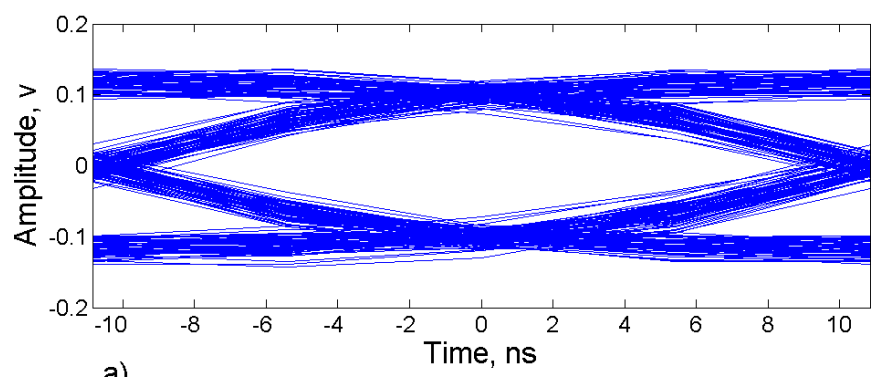

a)

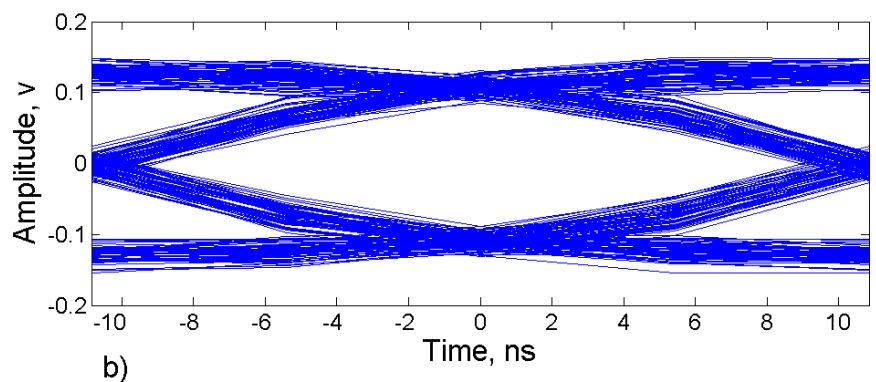

Figure 7. RF Transmit Signal Eye Diagrams. a) In-Phase Signal b) Quadrature Signal

\section{Conclusion}

A COTS RF and Optical SDR prototype was developed. The SDR consists of a COTS Xilinx development board with a COTS RF mezzanine card and a custom optical mezzanine card. The SDR currently supports SCPPM for the optical waveform and OQPSK modulation for the RF waveform. The electro optic subsystem uses the electrical SCPPM signal output from the SDR to modulate a laser. The optics subsystem sends the modulated light through an axicon and a Cassegrain telescope to be transmitted. The RF subsystem filters and up-converts the I and Q signals coming out of the SDR for transmission over the air. Initial testing shows that with a $50 \mathrm{~ns}$ slot clock, $\mathrm{K}_{\mathrm{s}}$ was 4.3 and $\mathrm{K}_{\mathrm{b}}$ was 0.00036 when using a high extinction ratio electro optic modulator. The RF OQPSK modulated signal had an EVM of $11.7 \%$ rms.

American Institute of Aeronautics and Astronautics 
Future developments will continue in order to increase the technology readiness level of the RF and optical SDR. Characterization of the optical transmitter system under varying background light conditions will occur. The RF and optical designs will be ported to an updated version of the Harris SDR which flew on the SCaN Test bed on the International Space Station in order to start development on a platform designed for space.

\section{Acknowledgments}

The authors would like to thank the NASA iROC team members for their work on the RF and Optical SDR prototype, including Dr. Sarah Tedder, Mr. Brian Vyhnalek, and Ms. Caitlyn Harrington. This work was performed under the NASA SCaN Program at the NASA Glenn Research Center.

\section{References}

${ }^{1}$ D. Raible, R. Romanofsky, J. Budinger, J. Nappier, A. Hylton, A. Swank, and A. Nerone. "On the Physical Realizability of Hybrid RF and Optical Communications Platforms for Deep Space Applications." AIAA International Communications Satellite Systems Conference, AIAA, San Diego, CA, 2014.

${ }^{2}$ A. Swank, E. Aretskin-Hariton, D. Le, O. Sands, A. Wroblewski. "Beaconless Pointing for Deep-Space Optical Communication.” AIAA International Communications Satellite Systems Conference, AIAA, Cleveland, OH, 2016.

${ }^{3}$ D. Boroson, B. Robinson, D. Murphy, D. Burianek, F. Khatri, J. Kovalik, Z. Sodnik, D. Cornwell. "Overview and Results of the Lunar Laser Communication Demonstration." Proceedings of the Free-Space Laser Communication and Atmospheric Propagation XXVI Conference, Vol. 8971, SPIE, 2014.

${ }^{4}$ B. Moison and J. Hamkins. "Coded Modulation for the Deep-Space Optical Channel: Serially Concatenated Pulse Position Modulation.” The Interplanetary Network Progress Report, vol. 42-161, May 2005.

${ }^{5}$ D. Zeleznikar, J. Nappier, and J. Downey. "Ka-Band Link Study for a Mars Hybrid RF/Optical Software Defined Radio." AIAA International Communications Satellite Systems Conference, AIAA, San Diego, CA, 2014.

${ }^{6}$ J. A. Downey, J. M. Downey, R. Reinhart, M. Evans, and D. Mortensen. "Bandwidth-Efficient Communication through 225 MHz Ka-band Relay Satellite Channel.” AIAA International Communications Satellite Systems Conference, AIAA, Cleveland, OH, 2016.

${ }^{7}$ S. Johnson, R. Reinhart, T. Kacpura. "CoNNeCT's Approach for the Development of Three Software Defined Radios for Space Application.” IEEE Aerospace Conference, Big Sky, Montana, 2012.

${ }^{8}$ J. Nappier, R. Tokars, and A. Wroblewski. "An Optical Receiver Post-Processing System for the Integrated Radio and Optical Communications Software Defined Radio Test Bed.” AIAA International Communications Satellite Systems Conference, AIAA, Cleveland, OH, 2016.

American Institute of Aeronautics and Astronautics 